\title{
Residual properties of free groups II
}

\section{Stephen J. Pride}

In this paper it is proved that non-abelian free groups are residually $\left(x, y \mid x^{m}=1, y^{n}=1, x^{k}=y^{h}\right) \quad$ if and only if $\min \{(m, k),(n, h)\}$ is greater than 1 , and not both of $(m, k)$ and $(n, h)$ are 2 (where 0 is taken as greater than any natural number). The proof makes use of a result, possibly of independent interest, concerning the existence of certain automorphisms of the free group of rank two. A useful criterion which enables one to prove that non-abelian free groups are residually $G$ for a large number of groups $G$ is also given.

\section{Introduction}

For unexplained concepts and notation the reader is referred to [5].

Let $A$ and $B$ be groups. $A$ is said to be residually $B$ if and only if for each non-identity element $a$ of $A$ there is a homomorphism $n$ which maps $A$ onto $B$ and is such that $\eta(a)$ is not the identity of $B$. If $A$ is a set of groups then $A$ is said to be residually $B$ if and only if each element $A$ of $A$ is residually $B$.

This paper reports on further developments in the program of studying residual properties of free groups. As is well-known (see [3]) non-abelian free groups are residually $B$ if and only if $F_{2}$ is residually $B$, where $F_{2}$ is a free group of rank two. Let $\{x, y\}$ be a generating set for $F_{2}$.

Received 20 March 1972. Communicated by li.F. Newman. Paper I in this series is not referred to in the present paper. The author thanks $\mathrm{Dr}$ if. $F$. Newman for his help in the preparation of this paper. 
In [1] Katz and Magnus showed that $F_{2}$ is residually $\left(x, y \mid x^{2}=1\right)$, and in [4] Poss proved that $F_{2}$ is residually $\left(x, y \mid x^{n}=1, y^{n}=1\right)$ if $n \geq 6$. In this paper a more general question is considered: for which of the groups

$$
\left(x, y \mid x^{m}=1, y^{n}=1, x^{k}=y^{h}\right)=\|m, n, k, h\|
$$

is $F_{2}$ residually $\|m, n, k, h\| ?$ (Here $m$ and $n$ are non-negative integers, and $k$ and $h$ are integers.) It is possible to adapt the proofs of the results of Katz and Magnus and of Poss to show that $F_{2}$ is residually $\|m, n, k, h\|$ for a large number of values of the quadruplet $(m, n, k, h)$, but a complete answer does not seem possible using [1] and [4].

THEOREM 1. The free group $F_{2}$ is residually $\|m, n, k, h\|$ if and only if $\min \{(m, k),(n, h)\}$ is greater than one, and not both of $(m, k)$ and $(n, h)$ are equal to two.

Here 0 should be taken to be greater than any other natural number.

The proof of Theorem I makes use of the following theorem, which will be proved in Section 2.

THEOREM 2. Let $w$ be a non-identity element of $F_{2}$. There is an automorphism, $\phi$, of $F_{2}$ (depending on $w$ ) such that $\phi(w)$ has the form

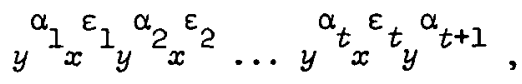

where $t \geq 1, \quad \alpha_{i}(1 \leq i \leq t+1)$ is an integer with $\left|\alpha_{i}\right| \leq 2$ and is non-zero except possibly if $i$ is equal to 1 or $t+1, \quad\left|\varepsilon_{i}\right|=1$ $(1 \leq i \leq t)$.

It should be remarked that in their proof that $F_{2}$ is residually $\| 2,0,0$, oll, Katz and Magnus [1] essentially obtain the following weak version of Theorem 1 :

if $w$ is a non-identity element of $F_{2}$, there is an automorphism, $\phi$, of $F_{2}$ (depending on $w$ ) such that $\phi(w)$ has the form 


$$
y^{\beta_{1}} \varepsilon_{1}^{\varepsilon_{1} \beta_{2} \varepsilon_{x}} \ldots y^{\beta_{s}}{ }_{x}^{\varepsilon_{s}}{ }_{y}^{\beta_{s+1}}
$$

where $s \geq 1, \beta_{i}(1 \leq i \leq s+1)$ is an integer, non-zero except possibly if $i$ equals 1 or $s+1,\left|\varepsilon_{i}\right|=1$

$(1 \leq i \leq s)$.

For the proof of Theorem 1 , it is no loss of generality to assume that $(n, h) \geq(m, k)$, since $\|m, n, k, h\|$ and $\|n, m, h, k\|$ are isomorphic. Suppose that $(n, h) \geq(m, k)>I$ and not both of $(n, h)$ and $(m, k)$ are equal to two. Let $N[m, n, k, h]$ be the normal subgroup of $F_{2}$ generated by $\left\{x^{m}, y^{n}, x^{k} y^{-h}\right\}$, so that $\|m, n, k, h\|$ is the factor group of $F_{2}$ by $N[m, n, k, h]$. If $w$ is a non-identity element of $F_{2}$ then by Theorem 2 there is an automorphism, $\phi$, of $F_{2}$ such that $\phi(w)$ has the form (*). It follows from Theorem 4.1 of [2] that $\phi(w)$ does not belong to $N[(m, k),(n, h), 0,0]$. Thus, if $\rho$ is the natural homomorphism of $F_{2}$ onto $\|m, n, k, h\|$ and $\pi$ is the homomorphism of $\|m, n, k, h\|$ onto $\|(m, k),(n, h), 0$, ol defined by

$$
\pi\left(g^{N}[m, n, k, h]\right)=g^{N}[(m, k),(n, h), 0,0]\left(g \in F_{2}\right),
$$

then $\pi \rho \phi(w)$ is not the identity of $\|(m, k),(n, h), 0$, oll. Thus $\rho \phi(w)$ is not the identity of $\|m, n, k, h\|$. This establishes that $F_{2}$ is residually $\|m, n, k, h\|$.

Now suppose that $(m, k)=1$ or $(m, k)=(n, h)=2$. Then $\|m, n, k, h\|$ is either cyclic or metabelian and so does not generate the variety of all groups. Consequently $F_{2}$ is not residually $\|m, n, k, h\|$

Before giving a proof of Theorem 2 some general remarks relating to the proof of the 'if' part of Theorem 1 will be made.

A normal subgroup, $N$, of $F_{2}$ is said to have the trivial intersection property (TI-property) if

$$
\bigcap_{\phi \in \operatorname{aut}\left(F_{2}\right)} \phi(N)=1,
$$

or equivalently, if $N$ contains no non-trivial characteristic subgroup of 
$F_{2}$. Clearly $F_{2}$ is residually $F_{2} / N$. Examples of normal subgroups with the TI-property are provided by the groups $N[p, q, 0,0]$ where $\min \{p, q\} \geq 2$ and not both of $p$ and $q$ are 2 . It can also be shown (for instance by using Theorem 2 [or the weak version of it due to Katz and Magnus] and Exercise 12, Section 4.4 of [2]) that if $r \geq 1$ and $|p|,|q|>1$ then the normal subgroup of $F_{2}$ generated by $\left\{y^{p} x^{p} y^{-r} x^{q}\right\}$ has the TI-property.

It is obvious that any normal subgroup of $F_{2}$ contained in a normal subgroup with the TI-property also has the TI-property. This gives a simple method for showing that $F_{2}$ is residually the group $G$.

CRITERION. Let $G$ be a group with presentation

$$
\left(x, y \mid r_{1}=1, r_{2}=1, \ldots\right)
$$

(where the number of relations can be finite or infinite). Then $F_{2}$ is residually $G$ if there is a normal subgroup, $N$, of $F_{2}$ with the TI-property such that

$$
\left\{r_{1}, r_{2}, \ldots\right\} \subseteq N
$$

It should be noticed that the proof of the 'if' part of Theorem $l$ is just an application of this criterion with $N=N[(m, k),(n, h), 0,0]$.

\section{Proof of Theorem 2}

As is well-known (see Theorem 4.2 of [2]) every non-identity element of $E_{2}$ is conjugate to an element of one of the following types:

$$
\begin{aligned}
& \text { (i) } x^{k}, k \neq 0 \text {; } \\
& \text { (ii) } y^{k}, k \neq 0 \text {; }
\end{aligned}
$$

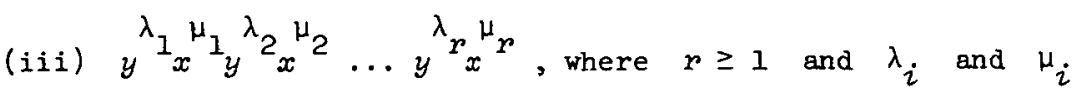

$$
\begin{aligned}
& (1 \leq i \leq r) \text { are non-zero integers. }
\end{aligned}
$$

It may therefore be assumed that $w$ is of type (i), (ii) or (iii).

The result is clear unless $\omega$ is of type (iii). To deal with this 
case it is convenient to introduce the concept of an ending of an element of $F_{2}$.

DEFINITION. Let $u$ be a non-identity element of $F_{2}$. The nonidentity element $v$ of $F_{2}$ is said to be an ending for $u$ if and only if there is an element $g$ of $F_{2}$ such that $u=g v$, where $g$ is either empty or has the property that if the first symbol of $v$ is $x$ or $x^{-1}$ ( $y$ or $\left.y^{-1}\right)$, then the last symbol of $g$ is $y$ or $y^{-1}\left(x\right.$ or $\left.x^{-1}\right)$.

Thus $y^{2} x$ is an ending for $x^{-2} y^{2} x$, whereas $y x$ is not. It is obvious that an element may have several endings. The phrases $i v$ is an ending for $u$ ' and ' $u$ ends in $v$ ' will be used synonymously.

Let $n$ be an integer with $n>0$, and let $\phi_{n}$ be the automorphism of $F_{2}$ defined by

$$
\begin{aligned}
& x \mapsto(y x)^{n} y^{2} x, \\
& y \mapsto\left((y x)^{n} y^{2} x\right)^{n}(y x)^{n} y .
\end{aligned}
$$

The following assertion will be proved by induction on $r$.

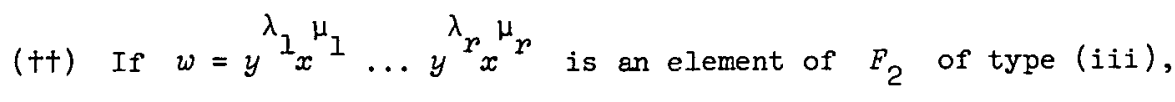
and $n$ is an integer satisfying

$$
n>\left|\mu_{i}\right|, i=1,2, \ldots, r,
$$

then $\phi_{n}(w)$ has the form $\left(^{*}\right)$ and ends in one of the following:

$$
\begin{aligned}
& x \\
& y\left((y x)^{n} y^{2} x\right)^{\mu_{r}} \quad\left(\text { if } \mu_{r}<0\right), \\
& \left(x^{-1} y^{-1}\right)^{n+1}\left((y x)^{n} y^{2} x\right)^{\mu_{r}} \quad\left(\text { if } \mu_{r}<0\right), \\
& y^{-1}\left(x^{-1} y^{-1}\right)^{n}\left(x^{-1} y^{-2}\left(x^{-1} y^{-1}\right)^{n}\right)^{n-\mu_{r}}, \\
& x^{-1} y^{-2}\left(x^{-1} y^{-1}\right)^{n-1}\left(x^{-1} y^{-2}\left(x^{-1} y^{-1}\right)^{n}\right)^{n+1-\mu_{r}} .
\end{aligned}
$$


It will be convenient in the following to denote the elements

$$
\begin{aligned}
& y\left((y x)^{n} y^{2} x\right)^{\mu}, \\
& \left(x^{-1} y^{-1}\right)^{n+1}\left((y x)^{n} y^{2} x\right)^{\mu}, \\
& y^{-1}\left(x^{-1} y^{-1}\right)^{n}\left(x^{-1} y^{-2}\left(x^{-1} y^{-1}\right)^{n}\right)^{n-\mu} r \\
& x^{-1} y^{-2}\left(x^{-1} y^{-1}\right)^{n-1}\left(x^{-1} y^{-2}\left(x^{-1} y^{-1}\right)^{n}\right)^{n+1-\mu} r,
\end{aligned}
$$

by $\gamma_{p}, \delta_{p}, o_{p}, \omega_{p}$ respectively. (Whenever $\gamma_{p}$ or $\delta_{r}$ is mentioned it is to be understood that $\mu_{p}<0$.) It will also be convenient to denote the element $(y x)^{n} y^{2} x$ by $p$, and the element $\left((y x)^{n} y^{2} x\right)^{n}(y x)^{n-1} y^{2} x$ by $q$. Notice that $p$ and $q$ are both of the form (*) and both have $y$ as first symbol and $x$ as last symbol.

If $\lambda$ and $\mu$ are non-zero integers, then it is not difficult to verify that $\Phi_{n}\left(y^{\lambda} x^{\mu}\right)$ is equal to
(1) $p^{n+1}(y x)^{n-1} y^{2} x q^{\lambda-1} p^{\mu-1}$ if $\lambda>0, \mu>0$,
(2) $p^{n}(y x)^{n} y p^{\mu}$ if $\lambda=1, \mu<0$,
(3) $p^{n+1}(y x)^{n-1} y^{2} x q^{\lambda-2} p^{n-1}(y x)^{n} y p^{\mu}$ if $\lambda>1, \mu<0$,
(4) $y^{-1}\left(x^{-1} y^{-1}\right)^{n}\left(p^{-1}\right)^{n-\mu}$ if $\lambda=-1$,
(5) $y^{-1}\left(x^{-1} y^{-1}\right)^{n}\left(p^{-1}\right)^{n-1}\left(q^{-1}\right)^{-\lambda-2} x^{-1} y^{-2}\left(x^{-1} y^{-1}\right)^{n-1}\left(p^{-1}\right)^{n+1-\mu}$ if $\lambda<-1$.

Using (1)-(5) it is easy to check that $(t+)$ holds when $r=1$.

Now assume that $r$ is greater than 1 . Let $w=y^{\lambda_{1}}{ }_{x}{ }^{\mu} \ldots y^{\lambda_{r}}{ }_{x}{ }_{r}$ be an element of type (iii), and let $n$ be an integer satisfying

$$
n>\left|\mu_{i}\right|, i=1,2, \ldots, r \text {. }
$$

Denote $y^{\lambda_{1}}{ }^{\mu}{ }_{1} \ldots y^{\lambda_{r-1}}{ }_{x}^{\mu_{p-1}}$ by $w_{1}$. 
By the induction hypothesis $\phi_{n}\left(w_{1}\right)$ has the form $\left(^{*}\right)$ and ends in one of $x, \gamma_{r-1}, \delta_{r-1}, \sigma_{r-1}, \omega_{r-1}$. If $\phi_{n}\left(w_{1}\right)$ ends in $x, \gamma_{r-1}$, or. $\delta_{r-1}$ then using (1)-(5) it can be shown, without too much difficulty that $\Phi_{n}(w)$ has the form $(*)$ and ends in one of $x, \gamma_{p}, \delta_{p}, \sigma_{r}, \omega_{r} ;$ when $\phi_{n}\left(\omega_{l}\right)$ ends in $\sigma_{r-1}$ or $\omega_{r-1}$ the verification is more complicated. Thus, suppose that $\phi_{n}\left(w_{1}\right)$ ends in $\sigma_{r-1}$. Then

$$
\phi_{n}\left(w_{1}\right)=g^{\sigma}{ }_{r-1}
$$

where $g$ is either empty or has $x$ or $x^{-1}$ as last symbol. Straightforward computations show

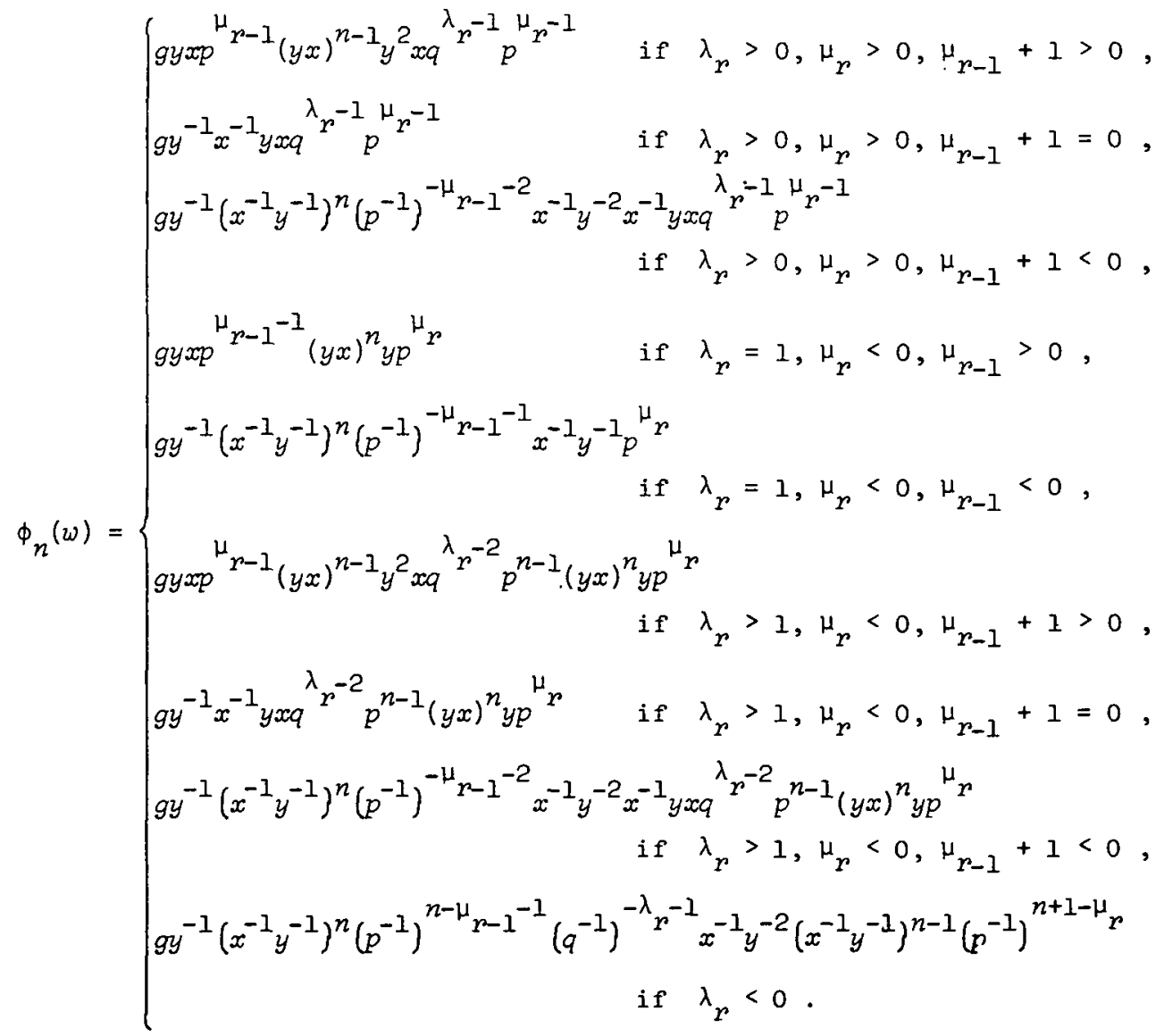


Hence $\phi_{n}(w)$ has the form $(*)$ and ends in one of $x, \gamma_{p}, \delta_{p}, \omega_{r}$.

The case when $\phi_{n}\left(w_{1}\right)$ ends in $\omega_{p-1}$ is similar to the case just considered, and details will be omitted.

\section{References}

[1] Robert A. Katz and Wilhelm Magnus, "Residual properties of free groups", Comm. Pure Appl. Math. 22 (1969), 1-13.

[2] Wi thelm Magnus, Abraham Karrass, Donald Solitar, Combinatorial group theory (Interscience [John Wiley \& Sons], New York, London, Sydney, 1966).

[3] Ada Peluso, "A residual property of free groups", Comm. Pure Appl. Math. $19(1966), 435-437$.

[4] Samuel Poss, "A residual property of free groups", Comm. Pure Appl. Math. 23 (1970), 749-756.

[5] Joseph J. Rotman, The theory of groups: An introduction (Allyn and Bacon, Boston, 1965).

Department of Mathematics, Institute of Advanced Studies, Australian National University, Canberra, ACT. 